\title{
FINITE ONE-RELATOR PRODUCTS OF TWO CYCLIC GROUPS WITH THE RELATOR OF ARBITRARY LENGTH
}

\author{
C. M. CAMPBELL, P. M. HEGGIE, E. F. ROBERTSON and R. M. THOMAS
}

(Received 7 September 1990; revised 1 February 1991)

Communicated by $\mathbf{H}$. Lausch

\begin{abstract}
In this paper we consider the groups $G=G(\alpha, n)$ defined by the presentations

$$
\left\langle a, b: a^{2}=b^{n}=a b^{-1} a b\left(a b a b^{-1}\right)^{\alpha-1} a b^{2} a b^{-2}=1\right\rangle .
$$

We derive a formula for $\left[G^{\prime}: G^{\prime \prime}\right]$ and determine the order of $G$ whenever $n \leq 7$. We show that $G$ is a finite soluble group if $n$ is odd, but that $G$ can be infinite when $n$ is even, $n \geq 8$. We also show that $G(6,10)$ is a finite insoluble group involving $\operatorname{PSU}(3,4)$, and that the group $H$ with presentation

$$
\left\langle a, b: a^{2}=b^{10}, a b^{-1} a b\left(a b a b^{-1}\right)^{5} a b^{2} a b^{-2}=1\right\rangle
$$
\end{abstract}

is a finite group of deficiency zero of order at least $114,967,210,176,000$.

1991 Mathematics subject classification (Amer. Math. Soc.): 20 F 05.

\section{Introduction}

In this paper, we consider certain one relator products of cyclic groups. In general, a one-relator product of groups $\left\{A_{i}: i \in I\right\}$ is a quotient $\left(* A_{i}\right) / N(R)$ where $\left(* A_{i}\right)$ is the free product of the groups $A_{i}(i \in I), R$ is a cyclically reduced word, and $N(R)$ is the normal closure of $R$ in $\left(* A_{i}\right)$. We are particularly interested here in the case where the $A_{i}$ are finite cyclic groups, especially in the case where $|I|=2$. In that case, if $A_{1}$ and $A_{2}$ are cyclic of orders $m$ and $n$ respectively, we have a presentation of the form

$$
\left\langle a, b: a^{m}=b^{n}=R(a, b)=1\right\rangle .
$$

(c) 1992 Australian Mathematical Society 0263-6115/92 $\$ A 2.00+0.00$ 
We shall normally assume that $R(a, b)$ is a word of the form

$$
a^{i(1)} b^{j(1)} \cdots a^{i(r)} b^{j(r)}
$$

where $r \geq 2,0<i(p)<m$ for all $p$ and $0<j(q)<n$ for all $q$. If $R=S^{k}$ is a proper power, then we have a generalized triangle group, and such a group is infinite if $(1 / m)+(1 / n)+(1 / k) \leq 1$ by [2]; this was proved independently and generalized in [19]. On a connected theme, it was shown in [32] that a group $X$ defined by a presentation of the form

$$
\left\langle x_{1}, x_{2}, \ldots, x_{m}: W\left(x_{1}, x_{2}\right)=W\left(x_{2}, x_{3}\right)=\cdots=W\left(x_{m}, x_{1}\right)=1\right\rangle
$$

with $m \geq 4$ is either cyclic or infinite. Given that result, it is natural to ask what happens if $m=2$ or $m=3$.

A group such as $X$ is an example of a cyclically presented group, and admits an automorphism of order dividing $m$ which permutes the $x_{i}$ in a cycle of length $m$. Such groups are of particular interest, in that many of the known finite groups of deficiency zero (that is finite groups with a presentation in which there is an equal number of generators and relators) are of this type; see [25] for a general survey. Forming the semi-direct product of $X$ with a cyclic group $\langle a\rangle$ of order $m$ yields the presentation

$$
\left\langle a, b: a^{m}=W\left(b, a^{-1} b a\right)=1\right\rangle,
$$

which can be rewritten in the form $\left\langle a, b: a^{m}=1, b^{n}=R(a, b)\right\rangle$, where $b$ has exponent sum zero in $R(a, b)$. If the corresponding group is finite, then the group defined by the presentation

$$
\left\langle a, b: a^{m}=b^{n}=R(a, b)=1\right\rangle
$$

is also finite. So we would like to know what happens when we have a presentation of the form $\left\langle a, b: a^{m}=1, b^{n}=R(a, b)\right\rangle$ or of the form $\left\langle a, b: a^{m}=b^{n}=R(a, b)=1\right\rangle$, where $R$ is not necessarily a proper power. In the latter case, we shall assume that $n>0$, and we shall normally assume that $b$ has exponent sum zero in $R(a, b)$. We concentrate on the case $m=2$.

If we have a presentation of the form $\left\langle a, b: a^{2}=b^{n}=a b^{i} a b^{-i}=1\right\rangle$, then the corresponding group is easily seen to be abelian of order $2 n$ or else infinite. On the other hand, the structure of a group defined by a presentation of the form $\left\langle a, b: a^{2}=b^{n}=a b^{i} a b^{j} a b^{k}=1\right\rangle$ was determined in [4], and further results on these, and the related deficiency zero groups defined by the presentations of the form $\left\langle a, b: a^{2}=a b^{i} a b^{j} a b^{k}=1\right\rangle$, may be found in $[8,9,10,11,31]$. The structure of the groups $G(n ; h, i, j, k)$ defined by the presentations

$$
\left\langle a, b: a^{2}=b^{n}=a b^{h} a b^{i} a b^{j} a b^{k}=1\right\rangle
$$


with $h+i+j+k=0$ and $h, i, j, k \in\{ \pm 1, \pm 2\}$ was determined in [14], and further results on such groups may be found in [13, 18]. Returning to the theme of cyclically presented groups, the Fibonacci group $F=F(2, n)$ is defined by the presentation

$$
\left\langle x_{1}, x_{2}, \ldots, x_{n}: x_{1} x_{2}=x_{3}, x_{2} x_{3}=x_{4}, \ldots, x_{n} x_{1}=x_{2}\right\rangle ;
$$

see [35] for a recent survey of these and related groups. It is known [17] that $F(2,1)$ and $F(2,2)$ are trivial, $F(2,3)$ is the quaternion group of order 8 , $F(2,4)$ is cyclic of order $5, F(2,5)$ is cyclic of order 11 and that $F(2,6)$ is infinite. Also, $F(2,7)$ is cyclic of order $29[3,16,21]$ and $F(2, n)$ is infinite for $n \geq 8$ [3, 27, 29]; see also [23, 34]. Forming a semi-direct product of $F$ with a cyclic group $\langle b\rangle$ of order $n$ permuting the generators cyclically yields the group $E=E(2, n)$ with presentation $\left\langle x, b: x b^{2}=b x^{2}, b^{n}=1\right\rangle$. If $n$ is even, then the relation $x^{n}=1$ also holds, since $\left(x^{-1} b^{2}\right)^{-1} b^{2}\left(x^{-1} b^{2}\right)=$ $b^{-2} x b^{2} x^{-1} b^{2}=b^{-1} x b^{2}=x^{2}$. If we add the relation $x^{n}=1$ in any case, and then the automorphism $a$ of order 2 interchanging $b$ and $x$, we get the group with presentation $\left\langle a, b: a^{2}=b^{n}=a b^{-1} a b a b^{2} a b^{-2}=1\right\rangle$. This has been shown $[12,14]$ to be metabelian of order $2 n g_{n}$ if $n$ is odd, where $\left(g_{n}\right)$ is the sequence of Lucas numbers defined by $g_{1}=1, g_{2}=3$ and $g_{n}=g_{n-1}+g_{n-2}$ for $n \geq 3$. (Since it contains $F(2, n)$ as a subgroup of index $2 n$ for $n$ even, the group is infinite if $n=2 m \geq 6$.)

In this paper, we consider the groups $G=G(\alpha, n)$ defined by the presentations

$$
\left\langle a, b: a^{2}=b^{n}=a b^{-1} a b\left(a b a b^{-1}\right)^{\alpha-1} a b^{2} a b^{-2}=1\right\rangle
$$

for $n \geq 1$ and $\alpha \geq 1$. Clearly $\left[G: G^{\prime}\right]=2 n$, and we show that $\left[G^{\prime}: G^{\prime \prime}\right]=$ $v_{n}(\alpha)$, where $v_{n}=v_{n}(\alpha)$ is defined by $v_{0}=0, v_{1}=1$ and

$$
v_{n}=\alpha v_{n-1}+v_{n-2}+1+(-1)^{n-1}
$$

for $n \geq 2$, and we point out some connections between these groups and the groups $F(2, n)$. We also investigate the structure of the groups $G(\alpha, n)$ for small values of $n$, and we have

TheOREM A. Let $G(\alpha, n)$ be the group defined by the presentation

$$
\left\langle a, b: a^{2}=b^{n}=a b^{-1} a b\left(a b a b^{-1}\right)^{\alpha-1} a b^{2} a b^{-2}=1\right\rangle,
$$

where $n \geq 1$ and $\alpha \geq 1$. Then

(i) $G(\alpha, 2)$ is dihedral of order $4 \alpha$;

(ii) $G(\alpha, 3)$ is metabelian of order $6 v_{3}(\alpha)=6\left(\alpha^{2}+3\right)$ if $\alpha \equiv 0,1$ or $2(\bmod 4)$, but has order $12 v_{3}(\alpha)=12\left(\alpha^{2}+3\right)$ and derived length 3 if $\alpha \equiv 3(\bmod 4)$; 
(iii) $G(\alpha, 4)$ is metabelian of order $8 v_{4}(\alpha)=8 \alpha\left(\alpha^{2}+4\right)$;

(iv) $G(\alpha, 5)$ is metabelian of order $10 v_{5}(\alpha)=10\left(\alpha^{4}+5 \alpha^{2}+5\right)$;

(v) $G(\alpha, 6)$ is metabelian of order $12 v_{6}(\alpha)=12 \alpha\left(\alpha^{2}+3\right)^{2}$ if $\alpha$ is even, is infinite of derived length 3 if $\alpha=1$, but has order $24((\alpha-1) / 2)^{3} v_{6}(\alpha)=$ $3(\alpha-1)^{3} \alpha\left(\alpha^{2}+3\right)^{2}$ and derived length 3 if $\alpha>1$ and $\alpha$ is odd;

(vi) $G(\alpha, 7)$ is metabelian of order $14 v_{7}(\alpha)=14\left(\alpha^{6}+7 \alpha^{4}+14 \alpha^{2}+7\right)$.

Part (i) of Theorem A is clear; we prove part (ii) in Section 3, parts (iii), (iv) and (vi) in Section 4, and part (v) in Section 5. The results given in Theorem $\mathrm{A}$ for $n$ odd are not atypical, as we also have

THEOREM B. Let $G=G(\alpha, n)$ be the group defined by the presentation

$$
\left\langle a, b: a^{2}=b^{n}=a b^{-1} a b\left(a b a b^{-1}\right)^{\alpha-1} a b^{2} a b^{-2}=1\right\rangle,
$$

where $n \geq 1$ and $\alpha \geq 1$. When $n$ is odd, $G$ is a finite soluble group of derived length at most 3. If, in addition, $\left(g_{n}, \alpha-1\right)=1$, then $G(\alpha, n)$ is a metabelian group of order $2 n v_{n}(\alpha)$.

Theorem B is proved in Section 4. However, not all the groups $G(\alpha, n)$ are finite; we show in Sections 6 and 7 that some of the groups $G(\alpha, 8)$ and $G(\alpha, 10)$ are infinite. Also, not all the finite groups $G(\alpha, n)$ are soluble; for example, the group $G(6,10)$ is a finite insoluble group involving $\operatorname{PSU}(3,4)$ (see Proposition 7.1). We summarize some results we have obtained concerning the groups $G(\alpha, 8)$ and $G(\alpha, 10)$ for small values of $\alpha$ in the following table.

TABLE 1

\begin{tabular}{|l|l|l|}
\hline$\alpha$ & $G(\alpha, 8)$ & $G(\alpha, 10)$ \\
\hline 2 & metabelian order 9,216 & metabelian order 67,240 \\
3 & metabelian order 75,504 & metabelian order $1,029,660$ \\
4 & derived length 3, order $11,197,440$ & metabelian order $9,302,480$ \\
5 & metabelian of order $1,691,280$ & metabelian order $57,002,500$ \\
6 & infinite soluble group & finite group involving $P S U(3,4)$ \\
7 & finite, derived length 4 or 5 & infinite group involving $H S$ \\
8 & metabelian order $37,914,624$ & \\
9 & metabelian order $84,321,360$ & \\
10 & infinite & \\
11 & infinite & \\
\hline
\end{tabular}


Here $H S$ denotes the Higman-Sims simple group of order $44,352,000$. Subsequently, Newman and O'Brien [30] have extended our results and have shown that $G(7,8)$ is soluble of order $2^{11} \cdot 3^{5} \cdot 7 \cdot 17^{2} \cdot 53$ and derived length 5 and that $G(6,10)$ has order $20|P S U(3,4)| v_{10}(6)=2^{9} \cdot 3^{2} \cdot 5^{3} \cdot 13 \cdot 1481^{2}$.

It is interesting to note that, if $G(\alpha, n)$ is finite, then certain related deficiency zero groups are also finite. For example, the group $H=H(\alpha, n)$ with presentation

$$
\left\langle a, b, z: a^{2}=b^{n}=z, a b^{-1} a b\left(a b a b^{-1}\right)^{\alpha-1} a b^{2} a b^{-2}=1\right\rangle
$$

is finite, since $z^{\alpha+1} \in H^{\prime} \cap Z(H) \leq \Phi(H)$, where $\Phi(H)$ denotes the Frattini subgroup of $H$. So, for example, $H(6,10)$ is a finite group of deficiency zero involving $P S U(3,4)$ of order at least $7|G(6,10)|$. (Subsequently, Newman and O'Brien [30] have shown that $H(6,10)$ has order $14|G(6,10)|=229,934,420,352,000$. Given that $G(7,8)$ has derived length 5 , we immediately see that $H(7,8)$ is a finite soluble group of deficiency zero with derived length at least 5 ; in fact, $H(7,8)$ has derived length precisely 5 and order $2^{4} \cdot 3 \cdot|G(7,8)|=2^{15} \cdot 3^{6} \cdot 7 \cdot 17^{2} \cdot 53$.)

The relationship explored in Section 4 between the groups $G(\alpha, n)$ and the Fibonacci groups $F(2, n)$ is of great help in determining which of the groups $G(\alpha, n)$ are finite. Also, the proofs of the above results show that $P S U(3,4)$ and $H S$ are homomorphic images of $F(2,10)$. It was pointed out in [26] that every finite 2-generator group $G$ (and therefore, in particular, every finite simple group-see [1,33]) is a homomorphic image of $F(2, n)$ for some value of $n$. However, while some results are known for specific groups as to which value of $n$ will suffice $[5,18,36]$, it does not seem to be easy, in general, to calculate the least value of $n$ that will suffice for a particular group $G$.

\section{The groups $G(\alpha, n)$}

Throughout this section, let $G=G(\alpha, n)$ be the group defined by the presentation

$$
\left\langle a, b: a^{2}=b^{n}=a b^{-1} a b\left(a b a b^{-1}\right)^{\alpha-1} a b^{2} a b^{-2}=1\right\rangle,
$$

where $n \geq 1$ and $\alpha \geq 1$. Let $c:=a b a$ and $N$ be the normal subgroup $\langle b, c\rangle$ of $G$. We see that $[G: N]=2$, and $N$ has presentation

$$
\left\langle b, c: b^{n}=c^{n}=c^{-1} b\left(c b^{-1}\right)^{\alpha-1} c^{2} b^{-2}=b^{-1} c\left(b c^{-1}\right)^{\alpha-1} b^{2} c^{-2}=1\right\rangle .
$$

Introduce a new generator $e:=c b^{-1}$, delete the generator $c=e b$, and then 
introduce the generator $d:=b e b^{-1}$, to get

$$
\begin{gathered}
\left\langle b, d, e: b^{n}=(e b)^{n}=1, b^{-1} d b=e, b^{-1} e b=e^{\alpha} d,\right. \\
\left.b^{-1} e b d^{-1} e^{-1}=d^{-1} e^{-1} b^{-1} e b\right\rangle .
\end{gathered}
$$

The last relation is equivalent to $e^{\alpha} d d^{-1} e^{-1}=d^{-1} e^{-1} e^{\alpha} d$ via the fourth, that is to $\left[d, e^{\alpha-1}\right]=1$, and so we have the presentation

$$
\left\langle b, d, e: b^{n}=(e b)^{n}=\left[d, e^{\alpha-1}\right]=1, b^{-1} d b=e, b^{-1} e b=e^{\alpha} d\right\rangle .
$$

So $G^{\prime}=\langle d, e\rangle$. If $\alpha=2$, the relation $[d, e]=1$ holds, and we have

Proposition 2.1. $G(2, n)$ is metabelian.

\section{We also have}

Proposition 2.2. If $G=G(\alpha, n)$, then $\left[G^{\prime}, G^{\prime \prime}\right]=v_{n}(\alpha)$.

In the proof of Proposition 2.2, we may assume that $G^{\prime}$ is abelian, so that $[d, e]=1$, and

$$
b^{-1} d b=e, \quad b^{-2} d b^{2}=d e^{\alpha}, \quad b^{-3} d b^{3}=e\left(d e^{\alpha}\right)^{\alpha}=d^{\alpha} e^{\alpha^{2}+1}, \quad \text { etc. }
$$

In general, we have $b^{-k} d b^{k}=d^{u_{k-1}} e^{u_{k}}$, where the sequence $\left(u_{n}\right)$ is defined by $u_{0}=0, u_{1}=1$ and $u_{n}=\alpha u_{n-1}+u_{n-2}$ for $n \geq 2$. The relation $(e b)^{n}=1$ may be replaced by $\left(d^{-1} b^{-1}\right)^{n}=1$, which, since $b^{n}=1$, may be rewritten as $d^{-1}\left(b^{-1} d^{-1} b\right)\left(b^{-2} d^{-1} b^{2}\right)\left(b^{-3} d^{-1} b^{3}\right) \cdots\left(b^{-(n-1)} d^{-1} b^{n-1}\right)=1$, which becomes $d^{-1} e^{-1}\left(d e^{\alpha}\right)^{-1}\left(d^{\alpha} e^{\alpha^{2}+1}\right)^{-1} \cdots\left(d^{u_{n-2}} e^{u_{n-1}}\right)^{-1}=1$. Let $\left(w_{n}\right)$ be the sequence defined by $w_{0}=0, w_{1}=1$ and $w_{n}=\alpha w_{n-1}+w_{n-2}+1$ for $n \geq 2$. Since $[d, e]=1$, we may collect terms and invert to get $d^{I} e^{J}=1$, where

$$
\begin{aligned}
& I:=1+u_{1}(\alpha)+u_{2}(\alpha)+u_{3}(\alpha)+\cdots+u_{n-2}(\alpha)=1+w_{n-2}(\alpha), \\
& J:=u_{1}(\alpha)+u_{2}(\alpha)+u_{3}(\alpha)+\cdots+u_{n-2}(\alpha)+u_{n-1}(\alpha)=w_{n-1}(\alpha)
\end{aligned}
$$

by [7, Corollary 9]. We now have the following presentation for the largest metabelian quotient of $N$

$$
\left\langle b, d, e: b^{-1} d b=e, b^{-1} e b=d e^{\alpha}, b^{n}=[d, e]=1, d^{I} e^{J}=1\right\rangle .
$$

Since $b^{-n} d b^{n}=d^{K} e^{L}$, where $K:=u_{n-1}(\alpha)$ and $L:=u_{n}(\alpha)$ the relation $b^{n}=1$ gives that $d^{K} e^{L}=d$, and we have

$$
\left\langle b, d, e: b^{-1} d b=e, b^{-1} e b=d e^{\alpha}, b^{n}=[d, e]=d^{I} e^{J}=d^{K-1} e^{L}=1\right\rangle .
$$


The normal abelian subgroup $G^{\prime}$ of index $n$ in $N$ is then seen to have order

$$
\left|\begin{array}{cc}
I & J \\
K-1 & L
\end{array}\right|=\left|\begin{array}{cc}
w_{n-2}(\alpha)+1 & w_{n-1}(\alpha) \\
u_{n-1}(\alpha)-1 & u_{n}(\alpha)
\end{array}\right|
$$

Replacing the first row of the determinant by the sum of the two rows, it follows from [7, Corollary 9 and Proposition 10] that the determinant has value $v_{n}(\alpha)$, and Proposition 2.2 follows.

\section{The groups $G(\alpha, 3)$}

In this section, we describe the structure of the groups $G=G(\alpha, 3)$. Here we have the presentation $\left\langle a, b: a^{2}=b^{3}=a b^{-1} a b\left(a b a b^{-1}\right)^{\alpha-1} a b^{2} a b^{-2}=1\right\rangle$ for $G$, and, as in Section 2, the presentation

$$
\left\langle b, d, e: b^{3}=(e b)^{3}=\left[d, e^{\alpha-1}\right]=1, b^{-1} d b=e, b^{-1} e b=e^{\alpha} d\right\rangle
$$

for the normal subgroup $N:=\langle b, a b a\rangle$ of index 2 in $G$. The relation $(e b)^{3}=1$ is equivalent to $d^{-1} b^{-1} d^{-1} b b^{-2} d^{-1} b^{2}=1$, that is $d^{-1} e^{-1}\left(e^{\alpha} d\right)^{-1}$ $=1$, which, given that $e^{\alpha-1}$ is central in $\langle d, e\rangle$, is equivalent to the relation $(e d)^{2}=e^{-(\alpha-1)}$. So $e^{\alpha} d=(e d)^{-2} e d=d^{-1} e^{-1}$, and we have the presentation

$$
\left\langle b, d, e: b^{3}=\left[d, e^{\alpha-1}\right]=1,(e d)^{2}=e^{-(\alpha-1)}, b^{-1} d b=e, b^{-1} e b=d^{-1} e^{-1}\right\rangle .
$$

We let $f$ denote $b^{-2} d b^{2}$, and we have the following presentation for $G^{\prime}=$ $\langle d, e, f\rangle$

$$
\begin{array}{r}
\left\langle d, e, f:\left[d, e^{\alpha-1}\right]=\left[e, f^{\alpha-1}\right]=\left[f, d^{\alpha-1}\right]=f e d=1,(e d)^{2}=e^{-(\alpha-1)},\right. \\
\left.(f e)^{2}=f^{-(\alpha-1)},(d f)^{2}=d^{-(\alpha-1)}\right\rangle .
\end{array}
$$

Delete the generator $f=d^{-1} e^{-1}$ to get

$$
\begin{aligned}
\left\langle d, e:\left[d, e^{\alpha-1}\right]=\left[e,(e d)^{\alpha-1}\right]=\left[e, d^{\alpha-1}\right]=1,(e d)^{2}=e^{-(\alpha-1)}\right. \\
\left.d^{-2}=\left(d^{-1} e^{-1}\right)^{-(\alpha-1)}, e^{-2}=d^{-(\alpha-1)}\right\rangle .
\end{aligned}
$$

Since $e^{-2}=d^{-(\alpha-1)}$, the relation $\left[e, d^{\alpha-1}\right]=1$ is redundant, and, since $(e d)^{2}=e^{-(\alpha-1)}$, we have that $\left[e d, e^{\alpha-1}\right]=1$, so that $\left[d, e^{\alpha-1}\right]=1$ is also redundant. Since $d^{-2}=\left(d^{-1} e^{-1}\right)^{-(\alpha-1)}$, we have that $\left[d,(e d)^{\alpha-1}\right]=1$, and so $\left[d(e d)^{-1},(e d)^{\alpha-1}\right]=1$, so that $\left[e,(e d)^{\alpha-1}\right]=1$ is redundant. We now have the presentation

$$
\left\langle d, e:(e d)^{2}=e^{-(\alpha-1)}, d^{-2}=\left(d^{-1} e^{-1}\right)^{-(\alpha-1)}, e^{-2}=d^{-(\alpha-1)}\right\rangle .
$$


Since $d^{\alpha-1}, e^{\alpha-1}$ and $(e d)^{\alpha-1}$ are central in $G^{\prime}, e^{-1} d e d^{-1}=e^{-2}(e d)^{2} d^{-2}$ is also ce al, and hence $z:=e^{-1} d e d^{-1}=d^{-1} e^{-1} d e$ is central. Now $(e d)^{2}=e(d e) d=e(e d z) d=e^{2} d^{2} z$, and so we have

$$
\begin{aligned}
&\left\langle d, e, z: z=d^{-1} e^{-1} d e, d^{2} z=e^{-(\alpha+1)}, d^{-2}=\left(d^{-1} e^{-1}\right)^{-(\alpha-1)},\right. \\
&\left.e^{-2}=d^{-(\alpha-1)}\right\rangle .
\end{aligned}
$$

As $d^{2}$ is central, we have that

$$
z^{2}=\left(d^{-1} e^{-1} d e\right)\left(e^{-1} d e d^{-1}\right)=d^{-1} e^{-1} d^{2} e d^{-1}=d^{-1} e^{-1} e d^{-1} d^{2}=1 .
$$

The relation $d^{-2}=\left(d^{-1} e^{-1}\right)^{-(\alpha-1)}$ is equivalent to $d^{-2}=(e d)^{(\alpha-1)}=$ $e^{\alpha-1} d^{\alpha-1} z^{(\alpha-1)(\alpha-2) / 2}$, so that we have

$$
\begin{aligned}
\left\langle d, e, z: z=d^{-1} e^{-1} d e, z^{2}=1, d^{2} z=e^{-(\alpha+1)}\right. \\
\left.d^{-(\alpha+1)}=e^{\alpha-1} z^{(\alpha-1)(\alpha-2) / 2}, e^{-2}=d^{-(\alpha-1)}\right\rangle .
\end{aligned}
$$

We may modify the fourth relation via the fifth to get

$$
\begin{array}{r}
\left\langle d, e, z: z=d^{-1} e^{-1} d e, z^{2}=1, d^{2} z=e^{-(\alpha+1)}, d^{-2}=e^{\alpha+1} z^{(\alpha-1)(\alpha-2) / 2},\right. \\
\left.e^{-2}=d^{-(\alpha-1)}\right\rangle,
\end{array}
$$

and then replace the fourth relation, using the second and third, by $z=$ $z^{(\alpha-1)(\alpha-2) / 2}$. If $\alpha \equiv 1$ or $\alpha \equiv 2(\bmod 4)$, we immediately have that $z=1$, and $G(\alpha, 3)$ is metabelian of order $6 v_{3}(\alpha)$. If $\alpha \equiv 0(\bmod 4)$, then $\alpha+1$ is odd, and, as $e^{-(\alpha+1)}=d^{2} z$ and $e^{2}$ are central, we have that $e$ is central, and so $z=1$, so that $G(\alpha, 3)$ is metabelian of order $6 v_{3}(\alpha)$. So assume that $\alpha \equiv 3(\bmod 4)$, in which case the relation $z=z^{(\alpha-1)(\alpha-2) / 2}$ is redundant via $z^{2}=1$. We now have

$$
\left\langle d, e, z: z=d^{-1} e^{-1} d e, z^{2}=1, d^{2} z=e^{-(\alpha+1)}, e^{-2}=d^{-(\alpha-1)}\right\rangle .
$$

Since $e^{-(\alpha+1)}=d^{2} z$, we have that $e^{-(\alpha+1)(\alpha-1) / 2}=\left(d^{2} z\right)^{(\alpha-1) / 2}=d^{\alpha-1} z^{(\alpha-1) / 2}$ $=e^{2} z$, so that $e^{\left(\alpha^{2}+3\right) / 2}=z$ and $e^{\alpha^{2}+3}=1$. Given this, $d^{-(\alpha-1)}=\left(d^{2}\right)^{-(\alpha-1) / 2}$ $=\left(e^{-(\alpha+1)} z\right)^{-(\alpha-1) / 2}=e^{\left(\alpha^{2}-1\right) / 2} z=e^{\alpha^{2}+1}=e^{-2}$, and the last relation is redundant. So we have

$$
\left\langle d, e, z: z=d^{-1} e^{-1} d e, e^{\left(\alpha^{2}+3\right) / 2}=z, z^{2}=1, d^{2} z=e^{-(\alpha+1)}\right\rangle .
$$

We replace the relation $d^{2} z=e^{-(\alpha+1)}$ by $d^{2}=e^{\left(\alpha^{2}+3\right) / 2-(\alpha+1)}=e^{\left(\alpha^{2}-2 \alpha+1\right) / 2}$; we then replace the relation $z^{2}=1$ by $e^{\alpha^{2}+3}=1$ to get

$$
\left\langle d, e, z: z=d^{-1} e^{-1} d e, e^{\left(\alpha^{2}+3\right) / 2}=z, e^{\alpha^{2}+3}=1, d^{2}=e^{(\alpha-1)^{2} / 2}\right\rangle .
$$


The relation $z=d^{-1} e^{-1} d e$ is equivalent to $d^{-1} e d=e z=e^{\left(\alpha^{2}+5\right) / 2}$. We now delete the redundant generator $z$ to get

$$
\left\langle d, e: d^{-1} e d=e^{\left(\alpha^{2}+5\right) / 2}, e^{\alpha^{2}+3}=1, d^{2}=e^{(\alpha-1)^{2} / 2}\right\rangle .
$$

Since $\alpha \equiv 3(\bmod 4)$, we have that

$$
\left(\frac{\alpha^{2}+5}{2}\right)^{2} \equiv 1 \text { and } \frac{(\alpha-1)^{2}}{2} \frac{\left(\alpha^{2}+5\right)}{2} \equiv \frac{(\alpha-1)^{2}}{2}\left(\bmod \alpha^{2}+3\right) \text {, }
$$

and so $M$ is metabelian of order $2\left(\alpha^{2}+3\right)=2 v_{3}(\alpha)$. This completes the proof of Theorem A (ii).

\section{The connection with the Fibonacci groups}

Let $G=G(\alpha, n)$. With presentation for $N$ as in Section 2, we add a new generator $y:=e^{\alpha-1}$ to get

$$
\left\langle b, d, e, y: b^{n}=(e b)^{n}=[d, y]=1, e^{\alpha-1}=y, b^{-1} d b=e, b^{-1} e b=y e d\right\rangle .
$$

Clearly $y$ is central in $G^{\prime}=\langle d, e\rangle$. Since $\left[G^{\prime}: G^{\prime \prime}\right]$ is finite by Proposition 2.2, $y^{i} \in G^{\prime \prime}$ for some $i \geq 1$, and then $y^{i} \in G^{\prime \prime} \cap Z\left(G^{\prime}\right)$. Thus $G^{\prime}$ is a stem extension of $G^{\prime} /\left\langle y^{i}\right\rangle$, and hence $G^{\prime}$ is finite if and only if $G^{\prime} /\left\langle y^{i}\right\rangle$ is finite; thus $G^{\prime}$ is finite if and only if $G^{\prime} /\langle y\rangle$ is finite. Since $\langle y\rangle$ is central in $G^{\prime}$, which is normal in $N$,

$$
Y:=\langle y\rangle^{N}=\left\langle y, b^{-1} y b, \ldots, b^{-(n-1)} y b^{n-1}\right\rangle
$$

is a finitely generated abelian group. So $G^{\prime}$ is finite if and only if $G^{\prime} / Y$ is finite, and hence $N$ is finite if and only if $N / Y$ is finite. So we have

Proposition 4.1. $G(\alpha, n)$ is finite if and only if the group $\bar{N}$ with presentation

$$
\left\langle\beta, \delta, \varepsilon: \beta^{n}=(\varepsilon \beta)^{n}=\varepsilon^{\alpha-1}=1, \beta^{-1} \delta \beta=\varepsilon, \beta^{-1} \varepsilon \beta=\varepsilon \delta\right\rangle
$$

is finite.

The relation $(\varepsilon \beta)^{n}=1$ is equivalent to $(\delta \beta)^{n}=1$ via $\beta^{-1} \delta \beta=\varepsilon$, and hence to $\left(\beta^{-1} \delta^{-1}\right)^{n}=1$. If we introduce $\gamma:=\delta^{-1}$ and $\eta:=\varepsilon^{-1}$, and then delete $\delta=\gamma^{-1}$ and $\varepsilon=\eta^{-1}$, we get the presentation

$$
\left\langle\beta, \gamma, \eta: \beta^{n}=\left(\beta^{-1} \gamma\right)^{n}=\eta^{\alpha-1}=1, \beta^{-1} \gamma \beta=\eta, \beta^{-1} \eta \beta=\gamma \eta\right\rangle .
$$


The relation $\eta^{\alpha-1}=1$ is equivalent to $\gamma^{\alpha-1}=1$ via $\beta^{-1} \gamma \beta=\eta$. Now use the relation $\beta^{-1} \gamma \beta=\eta$ to delete the generator $\eta$ and get

$$
\left\langle\beta, \gamma: \beta^{n}=\left(\beta^{-1} \gamma\right)^{n}=\gamma^{\alpha-1}=1, \beta^{-2} \gamma \beta^{2}=\gamma \beta^{-1} \gamma \beta\right\rangle .
$$

Introduce a new generator $\tau:=\gamma^{-1} \beta$, and then delete $\gamma=\beta \tau^{-1}$, to get

$$
\left\langle\beta, \tau: \beta^{n}=\tau^{n}=\left(\beta \tau^{-1}\right)^{\alpha-1}=1, \beta^{-1} \tau^{-1} \beta^{2}=\beta \tau^{-2} \beta\right\rangle .
$$

So we have proved part (i) of the following result.

Proposition 4.2. (i) $G(\alpha, n)$ is finite if and only if the group $\bar{N}$ with presentation

$$
\left\langle\beta, \tau: \beta^{n}=\tau^{n}=\left(\beta \tau^{-1}\right)^{\alpha-1}=1, \tau \beta^{2}=\beta \tau^{2}\right\rangle
$$

is finite,

(ii) if $\bar{N}$ has derived length $t$, then $G(\alpha, n)$ has derived length $t$ or $t+1$.

To prove (ii), note that, if $\bar{N}$ has derived length $t$, then $\bar{N}^{\prime}=\langle\delta, \varepsilon\rangle$ has derived length $t-1$, so that the subgroup $\langle d, e\rangle$ of $N^{\prime}$ has derived length $t-1$ or $t$. But $\langle d, e\rangle=G^{\prime}$, so that $G$ has derived length $t$ or $t+1$.

If $n$ is odd, we adjoin the automorphism $\theta$ of order 2 interchanging $\beta$ and $\tau$ to the presentation of Proposition 4.2 (i) to get the group $K$ with presentation

$$
\left\langle\theta, \beta: \theta^{2}=\beta^{n}=\left(\theta^{-1} \beta^{-1} \theta \beta\right)^{\alpha-1}=\theta \beta \theta \beta^{2} \theta \beta^{-2} \theta \beta^{-1}=1\right\rangle,
$$

which is a homomorphic image of the group $H$ with presentation

$$
\left\langle\theta, \beta: \theta^{2}=\beta^{n}=\theta \beta \theta \beta^{2} \theta \beta^{-2} \theta \beta^{-1}=1\right\rangle .
$$

Now $H^{\prime}$ is abelian of index $2 n$ in $H$ (for $n$ odd) by either [12] or [14], so that $\langle\delta, \varepsilon\rangle$ is an abelian subgroup of $N / Y$, and, using the same argument as in the proof of Proposition 4.2 (ii), we see that $\langle d, e\rangle$ is a metabelian subgroup of $N$. Since $G^{\prime}=\langle d, e\rangle$, we have proved the first part of Theorem B. Now [12] shows that $H^{\prime}$ is abelian of order $g_{n}$ by finding the presentation $\left\langle x, y: x^{f_{n-3}+2} y^{f_{n-2}-1}=x^{f_{n-2}-1} y^{f_{n-1}+1}=[x, y]=1\right\rangle$ for $H^{\prime}$, where $\left(f_{n}\right)$ is the sequence of Fibonacci numbers. Applying the same argument to $K$ gives the following presentation for $K^{\prime}$.

$$
\left\langle x, y: x^{f_{n-3}+2} y^{f_{n-2}-1}=x^{f_{n-2}-1} y^{f_{n-1}+1}=x^{\alpha-1}=y^{\alpha-1}=[x, y]=1\right\rangle .
$$

So, if $\left(g_{n}, \alpha-1\right)=1$, then $\left|K^{\prime}\right|=1$ and the proof of Theorem B is complete.

If $n=2 m$ is even, then, given $\beta^{n}=1$ and $\tau \beta^{2}=\beta \tau^{2}$, we have

$$
\begin{aligned}
\tau^{2 m} & =\left(\beta^{-1} \tau \beta^{2}\right)^{m}=\left(\beta^{-2} \beta \tau^{2} \tau^{-1} \beta^{2}\right)^{m} \\
& =\left(\beta^{-2} \tau \beta^{2} \tau^{-1} \beta^{2}\right)^{m}=\left(\tau^{-1} \beta^{2}\right)^{-1} \beta^{2 m}\left(\tau^{-1} \beta^{2}\right)=1,
\end{aligned}
$$

so that the relation $\tau^{n}=1$ is redundant in Proposition $4.2(\mathrm{i})$, and we have 
Proposition 4.3. If $n$ is even, then $G(\alpha, n)$ is finite if and only if the group $\bar{N}$ with presentation $\left\langle\beta, \tau: \beta^{n}=\left(\beta \tau^{-1}\right)^{\alpha-1}=1, \tau \beta^{2}=\beta \tau^{2}\right\rangle$ is finite.

The Fibonacci group $F(2, n)$ admits an automorphism permuting the generators in a cycle of length $n$. Forming the semi-direct product of $F(2, n)$ with the cyclic group of order $n$ acting on $F(2, n)$ in this way yields the group $E(2, n)$ with presentation $\left\langle b, t: t^{n}=1, t b^{2}=b t^{2}\right\rangle$. Proposition 4.2 then gives

Proposition 4.4. If $F(2, n)$ is finite, then $G(\alpha, n)$ is finite for all $\alpha$.

We can say rather more. Recall that $Y:=\langle y\rangle^{N} \leq G^{\prime \prime} \cap Z\left(G^{\prime}\right) \leq \Phi\left(G^{\prime}\right)$. So, if $\bar{G}^{\prime}:=G^{\prime} / Y$ is cyclic, then $G^{\prime} / \Phi\left(G^{\prime}\right)$, and hence $G^{\prime}$, is cyclic. Now, in the homomorphism from $E(2, n)$ onto $\bar{N}, F(2, n)$ maps onto $\bar{G}^{\prime}$; hence, if $F(2, n)$ is cyclic, then $G^{\prime}$ is cyclic. Since $F(2, n)$ is cyclic for $n=4,5$ or 7 , this gives

Proposition 4.5. If $n=4,5$ or 7 , then $G(\alpha, n)$ is metabelian for all $\alpha$.

Combining Propositions 2.2 and 4.5 yields Theorem A (iii), (iv) and (vi). Now let $E:=E(2, n)$ and $F:=F(2, n)$. Notice that $E^{\prime}=F$ and, if $\theta: E \rightarrow \bar{N}$ is the natural homomorphism, then the kernel $K$ of $\theta$ is contained in $E^{\prime}$. So $\bar{N}^{\prime} \cong E^{\prime} / K=F / K$ and $F / K$ has presentation

$$
\begin{array}{r}
\left\langle a_{1}, a_{2}, \ldots, a_{n}: a_{1} a_{2}=a_{3}, a_{2} a_{3}=a_{4}, \ldots, a_{n-2} a_{n-1}=a_{n}, a_{n-1} a_{n}=a_{1},\right. \\
\left.a_{n} a_{1}=a_{2}, a_{1}^{\alpha-1}=a_{2}^{\alpha-1}=\cdots=a_{n}^{\alpha-1}=a_{1} a_{2} \cdots a_{n}=1\right\rangle .
\end{array}
$$

For convenience, we replace each $a_{i}$ by $x_{n-i+1}^{-1}$ to get the presentation

$$
\begin{array}{r}
\left\langle x_{1}, x_{2}, \ldots, x_{n}: x_{2} x_{3}=x_{1}, x_{3} x_{4}=x_{2}, \ldots, x_{n-1} x_{n}=x_{n-2}, x_{n} x_{1}=x_{n-1},\right. \\
\left.x_{1} x_{2}=x_{n}, x_{1}^{\alpha-1}=x_{2}^{\alpha-1}=\cdots=x_{n}^{\alpha-1}=x_{1} x_{2} \cdots x_{n}=1\right\rangle .
\end{array}
$$

This leads to the following result, which strengthens Proposition 4.4.

Proposition 4.6. $G(\alpha, n)$ is finite if and only if the homomorphic image $M(\alpha, n)$ of $F(2, n)$ with presentation

$$
\left\langle x_{1}, x_{2}, \ldots, x_{n}: \prod_{j=1}^{n} x_{j}=x_{i}^{\alpha-1}=1, x_{i}=x_{i+1} x_{i+2}\right\rangle
$$


is finite. Moreover, if $M(\alpha, n)$ is soluble of derived length $t$, then $G(\alpha, n)$ is soluble of derived length $t+1$ or $t+2$. If $n$ is even, then $M(\alpha, n)$ has presentation $\left\langle x_{1}, x_{2}, \ldots, x_{n}: x_{i}^{\alpha-1}=1, x_{i}=x_{i+1} x_{i+2}\right\rangle$.

\section{The groups $G(\alpha, 6)$}

In this section, we consider the group $G:=G(\alpha, 6)$ with presentation

$$
\left\langle a, b: a^{2}=b^{6}=a b^{-1} a b\left(a b a b^{-1}\right)^{\alpha-1} a b^{2} a b^{-2}=1\right\rangle .
$$

By Proposition 4.3, $G$ is finite if and only if the group $\bar{N}$ with presentation

$$
\left\langle\beta, \tau: \beta^{6}=\left(\beta \tau^{-1}\right)^{\alpha-1}=1, \tau \beta^{2}=\beta \tau^{2}\right\rangle
$$

is finite. It is not difficult to check that $\bar{N}^{\prime}$ has presentation

$$
\begin{aligned}
& \left\langle a_{1}, a_{2}, a_{3}, a_{4}, a_{5}, a_{6}: a_{1}^{\alpha-1}=a_{2}^{\alpha-1}=a_{3}^{\alpha-1}=a_{4}^{\alpha-1}=a_{5}^{\alpha-1}=a_{6}^{\alpha-1}=1,\right. \\
& \left.\quad a_{1} a_{2}=a_{3}, a_{2} a_{3}=a_{4}, a_{3} a_{4}=a_{5}, a_{4} a_{5}=a_{6}, a_{5} a_{6}=a_{1}, a_{6} a_{1}=a_{2}\right\rangle .
\end{aligned}
$$

We may delete the generators $a_{3}, a_{4}, a_{6}$ and $a_{5}$ in turn to get

$$
\begin{aligned}
\left\langle a_{1}, a_{2}: a_{1}^{\alpha-1}=a_{2}^{\alpha-1}=\left(a_{1} a_{2}\right)^{\alpha-1}=\left(a_{1} a_{2}^{2}\right)^{\alpha-1}\right. & =\left(a_{1}^{2} a_{2}^{-1}\right)^{\alpha-1}=\left(a_{2} a_{1}^{-1}\right)^{\alpha-1} \\
& \left.=a_{1}^{-1} a_{2}^{2} a_{1} a_{2}^{2}=a_{1}^{2} a_{2} a_{1}^{2} a_{2}^{-1}=1\right\rangle .
\end{aligned}
$$

If $\alpha$ is even, so that $\alpha-1$ is odd, then the relations $a_{1}^{\alpha-1}=1$ and $a_{1}^{-1} a_{2}^{2} a_{1}=$ $a_{2}^{-2}$ give that $a_{2}^{2}=a_{1}^{-(\alpha-1)} a_{2}^{2} a_{1}^{\alpha-1}=a_{2}^{-2}$, and so we have that $a_{2}^{\alpha-1}=a_{2}^{4}=1$, and hence that $a_{2}=1$. By symmetry, $\bar{N}^{\prime}$ is trivial, and hence $\bar{N}$ is cyclic of order 6. So, with notation as in Section 4, we have that $N / Y$ is cyclic of order 6. Now $Y \leq\langle d, e\rangle=G^{\prime}$ and $\left[N: G^{\prime}\right]=6$, so that $Y=G^{\prime}$, and hence $G^{\prime}$ is abelian. Thus $G$ is metabelian of order $12 v_{6}(\alpha)$ by Proposition 2.2.

Let us now consider the case where $\alpha=2 t+1$ is odd. In this case, $N$ has the presentation

$$
\left\langle b, d, e: b^{6}=(e b)^{6}=\left[d, e^{2 t}\right]=1, b^{-1} d b=e, b^{-1} e b=e^{2 t+1} d\right\rangle
$$

as in Section 2. We may delete the generator $e=b^{-1} d b=b^{5} d b^{-5}$, and then rewrite the presentation as

$$
\left\langle b, d: b^{6}=(d b)^{6}=\left[d,\left(b^{5} d b^{-5}\right)^{2 t}\right]=1, b^{4} d b^{-4}=\left(b^{5} d b^{-5}\right)^{2 t+1} d\right\rangle .
$$

If $d_{i}:=b^{i} d b^{-i}(0 \leq i \leq 5)$, then $G^{\prime}=\left\langle d_{0}, d_{1}, d_{2}, d_{3}, d_{4}, d_{5}\right\rangle$ has presen- 
tation

$$
\begin{aligned}
& \left\langle d_{0}, d_{1}, d_{2}, d_{3}, d_{4}, d_{5}: d_{0} d_{1} d_{2} d_{3} d_{4} d_{5}=\left[d_{0}, d_{5}^{2 t}\right]\right. \\
& \quad=\left[d_{1}, d_{0}^{2 t}\right]=\left[d_{2}, d_{1}^{2 t}\right]=\left[d_{3}, d_{2}^{2 t}\right] \\
& \quad=\left[d_{4}, d_{3}^{2 t}\right]=\left[d_{5}, d_{4}^{2 t}\right]=1, d_{4}=d_{5}^{2 t+1} d_{0}, d_{5}=d_{0}^{2 t+1} d_{1}, \\
& \left.\quad d_{0}=d_{1}^{2 t+1} d_{2}, d_{1}=d_{2}^{2 t+1} d_{3}, d_{2}=d_{3}^{2 t+1} d_{4}, d_{3}=d_{4}^{2 t+1} d_{5}\right\rangle .
\end{aligned}
$$

We add new generators $z_{0}, z_{1}, \ldots, z_{5}$, where $z_{i}:=d_{i}^{2 t}$ for each $i$. Since $G^{\prime}=\left\langle d_{i+1}, d_{i}\right\rangle$ for any $i$, the relation $\left[d_{i+1}, d_{i}^{2 t}\right]=1$ simply expresses the fact that $z_{i}$ is central in $G^{\prime}$. We eliminate $d_{5}, d_{4}, d_{2}, d_{3}$ and $z_{5}$ in turn, and then simplify, to get

$$
\begin{aligned}
& \left\langle d_{0}, d_{1}, z_{0}, z_{1}, z_{2}, z_{3}, z_{4}: d_{0}^{2 t}=z_{0}, d_{1}^{2 t}=z_{1},\left(d_{1}^{-1} d_{0}\right)^{2 t}=z_{1}^{2 t} z_{2},\right. \\
& z_{1}^{t} z_{4}^{t}=z_{0} z_{3}, z_{0}^{t} z_{1}^{-1} z_{2}^{2 t} z_{3}^{t} z_{4}^{2 t+1}=z_{0}^{t+1} z_{1} z_{3}^{t+1} z_{4}=d_{0}^{-1} d_{1}^{2} d_{0} d_{1}^{2} z_{1} z_{2}^{-2} z_{4}^{-1} \\
& \left.=d_{1}^{-1} d_{0}^{2} d_{1} d_{0}^{2} z_{0} z_{1}^{-2} z_{3}^{-1}=1, z_{i} \text { central }(0 \leq i \leq 4)\right\rangle ;
\end{aligned}
$$

full details may be found in [6]. Since $d_{0}^{-1} d_{1}^{2} d_{0}=d_{1}^{-2} z_{1}^{-1} z_{2}^{2} z_{4}$ and $d_{1}^{-1} d_{0}^{2} d_{1}$ $=d_{0}^{-2} z_{0}^{-1} z_{1}^{2} z_{3}, A:=\left\langle d_{0}^{2}, d_{1}^{2}, z_{0}, z_{1}, z_{2}, z_{3}, z_{4}, G^{\prime \prime}\right\rangle$ is a normal subgroup of index 4 in $G^{\prime}$. We may calculate that $A$ has presentation

$$
\begin{gathered}
\left\langle u, v, w, x, c, z_{0}, z_{1}, z_{2}, z_{3}, z_{4}: u^{t}=v^{t}=z_{0}, w^{t}=x^{t}=z_{1},\right. \\
\left(w^{-1} u\right)^{t} c^{t}=\left(x^{-1} v\right)^{t} c^{-t}=z_{1}^{2 t} z_{2}, z_{1}^{t} z_{4}^{t}=z_{0} z_{3}, z_{0}^{t} z_{1}^{-1} z_{2}^{2 t} z_{3}^{t} z_{4}^{2 t+1} \\
=z_{0}^{t+1} z_{1} z_{3}^{t+1} z_{4}=1, w x=x u w u^{-1}=x v w v^{-1}=z_{1}^{-1} z_{2}^{2} z_{4}, \\
\left.v u=w u w^{-1} v=x u x^{-1} v=z_{0}^{-1} z_{1}^{2} z_{3}, c \text { central, } z_{i} \text { central }\right\rangle .
\end{gathered}
$$

It follows easily now that $A$ is abelian, and we then derive, after deleting $x$ and $v$, the presentation

$$
\begin{array}{r}
\left\langle u, w, c, z_{0}, z_{1}, z_{2}, z_{3}, z_{4}: u^{t}=z_{0}, z_{0}^{-t-2} z_{1}^{2 t} z_{3}^{t}=1, w^{t}=z_{1},\right. \\
z_{1}^{-t-2} z_{2}^{2 t} z_{4}^{t}=z_{0}^{-2} z_{1}^{4 t+2} z_{2}^{2}=z_{0}^{t} z_{1}^{-1} z_{2}^{2 t} z_{3}^{t} z_{4}^{2 t+1}=z_{0}^{t+1} z_{1} z_{3}^{t+1} z_{4}=1, \\
\left.c^{t}=z_{0}^{-1} z_{1}^{2 t+1} z_{2}, z_{1}^{t} z_{4}^{t}=z_{0} z_{3}, \quad A \text { abelian }\right\rangle .
\end{array}
$$

If $t=0$, then $z_{i}=1$ for each $i$ and $A$ is isomorphic to $C_{\infty} \times C_{\infty} \times C_{\infty}$; so assume that $t \neq 0$. If $Z:=\left\langle z_{0}, z_{1}, z_{2}, z_{3}, z_{4}\right\rangle$, then $A / Z$ is isomorphic to $C_{t} \times C_{t} \times C_{t}$, so that $A$ has order $t^{3}|Z|$, where $Z$ has presentation

$$
\begin{array}{r}
\left\langle z_{0}, z_{1}, z_{2}, z_{3}, z_{4}: z_{0}^{-t-2} z_{1}^{2 t} z_{3}^{t}=1, z_{1}^{-t-2} z_{2}^{2 t} z_{4}^{t}=1, z_{0}^{-2} z_{1}^{4 t+2} z_{2}^{2}=1,\right. \\
\left.z_{1}^{t} z_{4}^{t}=z_{0} z_{3}, z_{0}^{t} z_{1}^{-1} z_{2}^{2 t} z_{3}^{t} z_{4}^{2 t+1}=z_{0}^{t+1} z_{1} z_{3}^{t+1} z_{4}=1, \quad Z \text { abelian }\right\rangle .
\end{array}
$$


Since $\left[G^{\prime}: A\right]=4$, we see that $G^{\prime}$ has order $4 t^{3}|Z|$, which a routine, but tedious calculation, shows to be $24((\alpha-1) / 2)^{3} v_{6}(\alpha)$ as required; again, the details may be found in [6].

\section{The groups $G(\alpha, 8)$}

In this section, we mention some results about the groups $G(\alpha, 8)$. Some of these results were originally obtained by using a computer, and have subsequently been provided with hand proofs, and some still rely on the computer proofs. In general, we used a Todd-Coxeter program, to which the third author has added a Reidemeister-Schreier routine based on [20] and the Tietze transformation program described in [22].

The group $G(2,8)$ is metabelian of order $16 v_{8}(2)=9,216$ by Propositions 2.1 and 2.2. The group $G(3,8)$ is metabelian of order $16 v_{8}(3)=$ 75,504 and $G(5,8)$ is metabelian of order $16 v_{8}(5)=1,691,280$; this may be easily verified by means of Reidemeister-Schreier and Tietze transformation programs, and hand proofs are given in [6]. The group $G(4,8)$ is not metabelian, however, as $G(4,8)^{\prime \prime}$ is elementary abelian of order 27 , so that $G(4,8)$ has derived length 3 and order $16.27 . v_{8}(4)=11,197,440$; again, this may be verified using the programs mentioned above.

In contrast to the situation with $n \leq 7$, the groups $G(\alpha, 8)$ are not necessarily finite. For example, if $\alpha=6$, we have the group $G(6,8)$ defined by the presentation

$$
\left\langle a, b: a^{2}=b^{8}=a b^{-1} a b\left(a b a b^{-1}\right)^{5} a b^{2} a b^{-2}=1\right\rangle .
$$

We give a hand proof in [6] that $G(6,8)$ is infinite, though one can readily verify this using Proposition 4.3 and the computer programs mentioned above, which show that $\bar{N}$ is soluble of derived length 4 with derived factors $C_{8}, C_{5},\left(C_{2}\right)^{4}$ and $\left(C_{\infty}\right)^{5}$. Newman and O'Brien [30] have since pushed this further; they deduce that $G(6,8)$ has derived length 5 and that it has a polycyclic series with 5 infinite sections. Note that, by Proposition 4.4 , this result gives yet another proof that $F(2,8)$ is infinite.

If $\alpha=7$, then $\bar{N}$ can be shown to have derived length 4 with derived factors $C_{8}, C_{3} \times C_{3}, C_{3}$ and $\left(C_{2}\right)^{6}$; so $G(7,8)$ has derived length 4 or 5 by Proposition 4.2 (ii); however, as we mentioned in the introduction, Newman and O'Brien [30] have since shown that $G(7,8)$ has derived length 5 , and full details of the computational techniques are included in their paper. On the other hand, if $\alpha=8$ or $\alpha=9$, then Todd-Coxeter shows that $\bar{N}$ is cyclic of order 8 in each case, so that $G(8,8)$ and $G(9,8)$ are metabelian of orders $16 v_{8}(8)=37,914,624$ and $16 v_{8}(9)=84,321,360$ respectively. We may 
use similar computational techniques to show that the groups $G(10,8)$ and $G(11,8)$ are infinite.

\section{The groups $G(\alpha, 10)$}

In this section we mention some rather surprising results concerning the groups $G(\alpha, 10)$, and show that certain of these groups are insoluble. Now, by Proposition 4.3, $G(\alpha, 10)$ is finite if and only if the group $\bar{N}$ with presentation $\left\langle\beta, \tau: \beta^{10}=\left(\beta \tau^{-1}\right)^{\alpha-1}=1, \tau \beta^{2}=\beta \tau^{2}\right\rangle$ is finite. If $\alpha=2,3,4$ or 5 , it is reasonably easy to check that $\bar{N}$ is cyclic of order 10 . However, if $\alpha=6$, then Reidemeister-Schreier yields the following presentation for $\bar{N}^{\prime}$ :

$$
\begin{aligned}
\left\langle x, y: x^{5}=\right. & y^{5}=(x y)^{5}=\left(x y^{-1}\right)^{5}=\left(x^{2} y\right)^{5}=\left(x y^{-2}\right)^{5} \\
= & \left(x^{2} y x y\right)^{5}=\left(x y^{-1} x y^{-2}\right)^{5} \\
= & \left(x y^{2} x y^{-2}\right)^{5}=\left(x y x^{-1} y^{2} x^{-1} y x y^{-2}\right)^{5}=1, \\
& x^{2} y x y^{2} x^{-1} y x y^{-1} x y^{-1} x y x^{-1} y^{2} x y=1, \\
& \left.\quad x^{2} y x^{2} y^{-1} x y^{-2} x y x^{-1} y^{2} x^{-1} y x y^{-2} x y^{-1}=1\right\rangle .
\end{aligned}
$$

A coset enumeration shows that $\bar{N}^{\prime}$ has order 62,400 , which is the same as that of $P S U(3,4)$; it follows immediately from [24] that $\bar{N}^{\prime}$ is isomorphic to $\operatorname{PSU}(3,4)$. So we have

Proposition 7.1. If $\alpha=6, n=10$, then $\bar{N}$ is an extension of $\operatorname{PSU}(3,4)$ by $C_{10}$. In particular, $P S U(3,4)$ is a homomorphic image of the Fibonacci group $F(2,10)$, and $G(6,10)$ is a finite insoluble group involving $P S U(3,4)$.

We can relate this presentation of $\operatorname{PSU}(3,4)$ to previously obtained presentations, since a computer calculation shows that $\bar{N}^{\prime}=\langle u, v\rangle$, where $u:=$ $y^{-1} x^{-1} y^{-2} x y^{-1} x^{-2} y^{-2} x y^{-1} x^{-1} y x^{-1} y^{3} x^{-1}$ and $v:=y x^{2} y$, and $\{u, v\}$ is a minimal generating pair for $\operatorname{PSU}(3,4)$ satisfying the relations $u^{2}=v^{3}=$ $(u v)^{15}=[u, v]^{5}=\left((u v)^{3}\left(u v^{-1}\right)^{3}\right)^{3}=\left(u v^{-1}(u v)^{5}\right)^{4}=1$ as detailed in [28]. Further details about $G(6,10)$ are provided in [30].

If we repeat the above computations with $\alpha=7$, we again get that $\bar{N}$ is an extension of a perfect group by $C_{10}$. This time, computer calculations show that there is a group $T$ of order $44,352,000$ which is a homomorphic image of $\bar{N}^{\prime}$, and $T$ acts as a permutation group on the cosets of a subgroup $\bar{K}$ of index 100 in $\bar{N}^{\prime}$. We have verified using Cayley [15] that $T$ is the HigmanSims simple group $H S$; however, the subgroup $\bar{K}$ has $\bar{K} / \bar{K}^{\prime}$ isomorphic to $C_{\infty}$, so that $G(7,10)$ is infinite. 


\section{Acknowledgments}

The authors would like to thank Alexander Wegner for his help with the computing results mentioned in this paper. The authors are grateful to Mike Newman and Eamonn O'Brien for their careful reading of this paper and for their helpful comments. The fourth author would also like to thank Hilary Craig for all her help and encouragement.

\section{References}

[1] M. Aschbacher and R. Guralnick, 'Some applications of the first cohomology group', $J$. Algebra 90 (1984), 446-460.

[2] G. Baumslag, J. W. Morgan and P. B. Shalen, 'Generalized triangle groups', Math. Proc. Camb. Phil. Soc. 102 (1987), 25-31.

[3] A. M. Brunner, 'The determination of Fibonacci groups', Bull. Austral. Math. Soc. 11 (1974), 11-14.

[4] C. M. Campbell, H. S. M. Coxeter and E. F. Robertson, 'Some families of finite groups having two generators and two relations', Proc. Roy. Soc. London 357A (1977), 423-438.

[5] C. M. Campbell, H. Doostie and E. F. Robertson, 'Fibonacci length of generating pairs in groups', in: Applications of Fibonacci numbers (eds. G. E. Bergum, A. N. Philippou and A. F. Horadam) (Kluwer, 1990), pp. 27-35.

[6] C. M. Campbell, P. M. Heggie, E. F. Robertson and R. M. Thomas, One-relator products of cyclic groups and Fibonacci-like sequences (Technical Report 35, Department of Computing Studies, University of Leicester, April 1990).

[7] C. M. Campbell, P. M. Heggie, E. F. Robertson and R. M. Thomas, 'One-relator products of cyclic groups and Fibonacci-like sequences', in: Applications of Fibonacci numbers (eds. G. E. Bergum, A. N. Phillippou and A. F. Horadam) (Kluwer, 1991), pp. 63-68.

[8] C. M. Campbell and E. F. Robertson, 'Classes of groups related to $F^{a, b, c}$, Proc. Roy. Soc. Edinburgh 78A (1978), 209-218.

[9] C. M. Campbell and E. F. Robertson, 'On 2-generator 2-relation soluble groups', Proc. Edinburgh Math. Soc. 23 (1980), 269-273.

[10] C. M. Campbell and E. F. Robertson, 'Groups related to $F^{a, b, c}$ involving Fibonacci numbers', in: The geometric vein (eds. C. Davis, B. Grünbaum and F. A. Sherk) (SpringerVerlag, 1982), pp. 569-576.

[11] C. M. Campbell and E. F. Robertson, 'On the $F^{a, b, c}$ conjecture', Mitt. Math. Sem. Giessen 164 (1984), 25-36.

[12] C. M. Campbell, E. F. Robertson and R. M. Thomas, 'Fibonacci numbers; and groups', in: Applications of Fibonacci numbers (eds. A. F. Horadam, A. N. Philippou and G. E. Bergum) (D. Reidel, 1987), pp. 45-59.

[13] C. M. Campbell, E. F. Robertson and R. M. Thomas, 'On groups related to Fibonacci groups', in: Group theory (eds. K. N. Cheng and Y. K. Leong) (Walter de Gruyter \& Co., 1989), pp. 323-331.

[14] C. M. Campbell and R. M. Thomas, 'On $(2, n)$-groups related to Fibonacci groups', Israel J. Math. 58 (1987), 370-380.

[15] J. J. Cannon, 'An introduction to the group theory language Cayley', in: Computational group theory (ed. M. D. Atkinson) (Academic Press, 1984), pp. 145-183.

[16] C. P. Chalk and D. L. Johnson, 'The Fibonacci groups II', Proc. Roy. Soc. Edinburgh 77A (1977), 79-86. 
[17] J. H. Conway et al., 'Solution to advanced problem 5327', Amer. Math. Monthly 74 (1967), 91-93.

[18] H. Doostie, Fibonacci-type sequences and classes of groups (Ph. D. Thesis, University of St Andrews, 1988).

[19] B. Fine, J. Howie and G. Rosenberger, 'One relator quotients and free products of cyclics', Proc. Amer. Math. Soc. 102 (1988), 249-254.

[20] G. Havas, 'A Reidemeister-Schreier program', in: Proc. Second Intern. Conf. Theory of Groups, Canberra 1973 (ed. M. F. Newman) (Lecture Notes in Mathematics 372, Springer-Verlag, 1974), pp. 347-356.

[21] G. Havas, 'Computer aided determination of a Fibonacci group', Bull. Austral. Math. Soc. 15 (1976), 297-305.

[22] G. Havas, P. E. Kenne, J. S. Richardson and E. F. Robertson, 'A Tietze transformation program', in: Computational group theory (ed. M. D. Atkinson) (Academic Press, 1984), pp. $69-73$.

[23] H. Helling, A. C. Kim and J. L. Mennicke, 'On Fibonacci groups', preprint.

[24] D. F. Holt and W. Plesken, Perfect groups (Oxford University Press, 1989).

[25] D. L. Johnson and E. F. Robertson, 'Finite groups of deficiency zero', in: Homological Group Theory (ed. C. T. C. Wall) (London Math. Soc. Lecture Note Series 36, Cambridge University Press, 1979) pp. 275-289.

[26] D. L. Johnson, J. W. Wamsley and D. Wright, 'The Fibonacci groups', Proc. London Math. Soc. 29 (1974), 577-592.

[27] R. C. Lyndon, 'On a family of infinite groups introduced by Conway', unpublished.

[28] J. McKay and K-C. Young, 'The nonabelian simple groups $G,|G|<10^{6}$-minimal generating pairs', Math. Comp. 33 (1979), 812-814.

[29] M. F. Newman, 'Proving a group infinite', Archiv. Math. 54 (1990), 209-211.

[30] M. F. Newman and E. A. O'Brien, 'A computer-aided analysis of some finitely-presented groups', J. Austral. Math. Soc.

[31] M. Perkel, 'Groups of type $F^{a, b,-c}$, Israel J. Math. 52 (1985), 167-176.

[32] S. J. Pride, 'Groups with presentations in which each defining relator involves exactly two generators', J. London Math. Soc. 36 (1987), 245-256.

[33] R. Steinberg, 'Generators for simple groups', Canad. J. Math. 14 (1962), 277-283.

[34] R. M. Thomas, 'The Fibonacci groups $F(2,2 m)$ ', Bull. London Math. Soc. 21 (1989), 463-465.

[35] R. M. Thomas, 'The Fibonacci groups revisited', in: Proceedings of Groups-St. Andrews 1989, vol. 2, (eds. C. M. Campbell and E. F. Robertson) (London Math. Soc. Lecture Note Series 160, Cambridge University Press, 1991) pp. 445-454.

[36] H. J. Wilcox, 'Fibonacci sequences of period $n$ in groups', Fibonacci Quart. 24 (1986), 356-361.

C. M. Campbell, P. M. Heggie and

R. M. Thomas

E. F. Robertson

Mathematical Institute

University of St Andrews

St Andrews KY16 9SS

Scotland

Department of Computing Studies University of Leicester Leicester LE1 7RH England 